\title{
Operation of the Information Monitoring System for the Optimization of the Processing Time and the Dissemination of Financial Data
}

\author{
Hanen Khanchel ${ }^{1}$, Mohamed Afif Lakhoua ${ }^{2}$ \& Karim Ben Kahla ${ }^{3}$ \\ ${ }^{1}$ Institute of higher commercial studies of Carthage, Tunisia \\ ${ }^{2}$ Higher Institute of management, Bardo, 2000, Tunisia \\ ${ }^{3}$ High Business School, Campus of Manouba, 2010, Manouba, Tunisia \\ Correspondence: Mohamed Afif Lakhoua, Higher Institute of management, Bardo, 2000, Tunisia
}

Received: August 15, 2017

Accepted: August 29, 2018

Online Published: September 18, 2018

doi:10.5430/bmr.v7n3p1

URL: https://doi.org/10.5430/bmr.v7n3p1

\begin{abstract}
In this paper, we propose the concept of "information monitoring system "; we indicate its usefulness in the process of informative anticipatory system, in the face of the problem of financial information overload caused by the use of ICT. We present a grid of analysis of the accounting process in an indebted company. The concept is useful when the information intelligence is oriented "exploitation of the information anticipatory" for the anticipation, these being embedded in large financial data. Our experimentation on the problem studied will show us that these tools are effective to optimize the time of processing and dissemination of financial information for decision-making.
\end{abstract}

Keywords: IMS, optimization process, dissemination time, financial data, accounting process

\section{Introduction}

The analysis of the literature reveals a focus on the ability of individuals to deal with information overload (Freitas, P.; and al.; 2008). The issue of time to process a task is little discussed. This seems to us to be an insufficient approach insofar as ICT reconstitute the temporal question in the firm (Delengaigne, X, 2014). Instantaneousness, reactivity, acceleration of processes, has consequence on the time an employee has to respond to a solicitation, to perform a task. Consequently, the temporal aspect of information overload seems to be decisive in the context of the generalization of ICT (Deiss, J., 2015). Indeed, there is a difficulty in managing the amount of information engendered by electronic exchange. The use of emails thus represents one of the cause of overflow with the time spent sorting and processing the information (Griffin, C. and al., 2014). Being overwhelmed by this large volume of information is likely to contribute to the feeling of work overload (Lesca, N. and al. 2008). We can conclude that there is a set of factors that vary from one system to another (Lakhoua M.N., 2018). These factors may change depending on organizations. They may be more or less flexible or constraining. This combined set of factors is likely to influence the relationship between managers' spheres of life. But as argued by Emilie Vayre, (2016), it remains difficult or even unnecessary to measure the weight of each of these determinants, for it is their articulation that makes sense. Information anticipatory is the monitoring of the publication of information relevant to a subject by means of research, processing and dissemination activities (De Montmollin, P. 2013); It is used for personal use: keep abreast of current business, a university research subject or in a professional manner: with a view to using the information collected by persons for whom it is useful; May represent a competitive advantage (Lesca, H., Lesca, N., 2014). In this context, the purpose of informational intelligence is to optimize decision-making time (Basset, H. 2013). This discipline is based on the observation and analysis of the scientific, technological and technological environment and the present and future economic impacts to infer threats and opportunities for development (De Vincenzo et al., 2016). Gallo and al. (2016) propose the Business Intelligence and Analytics Platforms. Business analytics (BA) is the practice of iterative, methodical exploration of an organization's data with emphasis on statistical analysis. Business analytics is used by companies committed to data-driven decision making. Lesca, H. and al. (2011) propose the Information Monitoring System (IMS) to enable the organization to act in a timely manner, at the right time, with maximum efficiency and minimum resources, with the aim of contributing to its sustainable competitiveness. However, this method, which is relevant in the case of previously selected information, is defeated by the absence of appropriate information overload management tools (Drevon, E. and al., 2016). This problem is felt in the phase of interpretation of the information and in particular in the preparation of the session of collective 
creation of meaning which is at the center of this method. Collective meaning is defined as a group of people [who] voluntarily agree to share (collectively) their ability to detect events, to speak about them, to interpret them together and to draw useful lessons from them For action (Almeida, F. C.; and al. , 2016). To do this, it is necessary to select from the many information (often 'raw data') collected by the company of the information between which it is possible to establish links. This will be called "monitoring information". In the face of information overload, how to select neighboring information? How can one measure the proximity (proximity) between two pieces of information? We will focus on textual numerical information (Sadok, M., Lesca, H. 2009). We will define in the first part the context of this research as well as the problem. We will present in a second part the prototype developed to answer this problem and we will describe the experimentation of this tool as part of a strategic intelligence project. We draw some lessons from the conclusion.

\section{Methodological Approach}

The Process of Anticipatory monitoring and Collective Creation of Sens is defined as "a collective and proactive process by which members of the company use information of an anticipatory and relevant nature concerning their external environment and the changes that may occur therein" (Lesca, N. and al., 2008). By anticipatory information we designate information useful for designing anticipations of events that are likely to influence the future of the company the process is illustrated in figure 1.

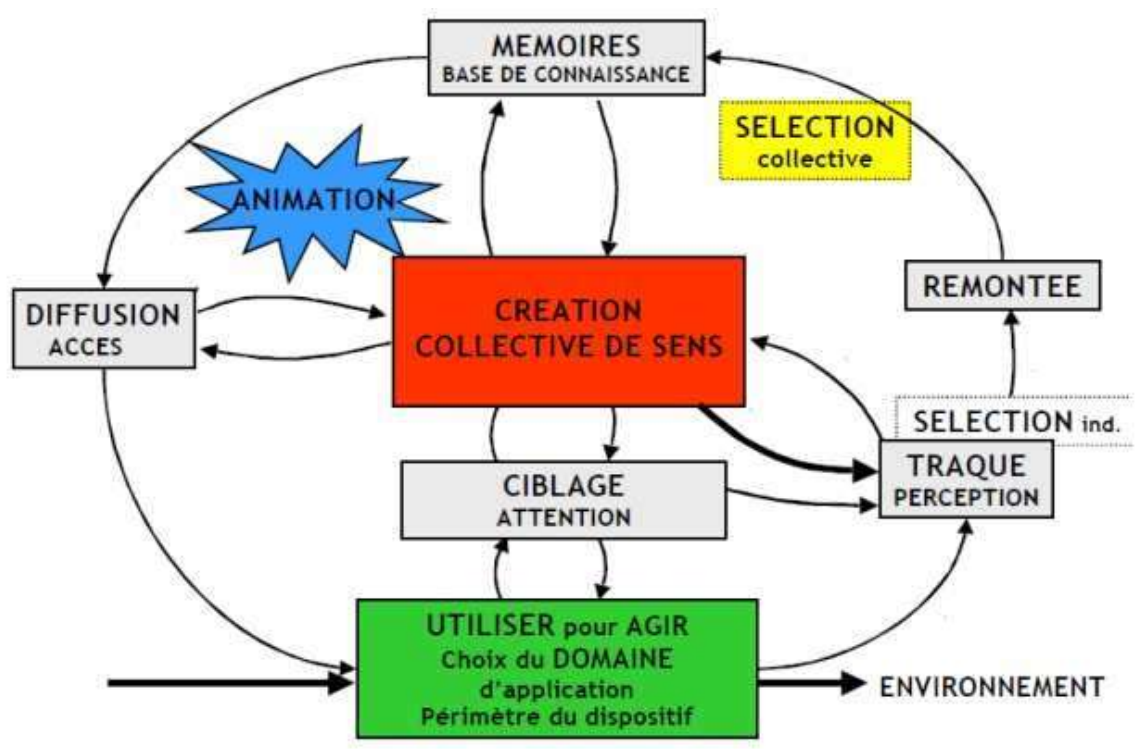

Figure 1. Generic Process of the Information Monitoring System

A collective work session is organized to create "added meaning" and knowledge from information that acts as a stimulus. The interactions between the participants and the various (tacit and formal) memories of the company will help to make "sense" emerge. A session of collective creation of meaning results in the formulation of temporary conclusions (plausible hypotheses) that can lead to actual actions (Blanco, S. Lesca, N. 2003).

To help companies create collective meaning, we propose a method called "Puzzle" (Lesczczynska, D. et Lesca, H. 2004). The idea at the origin is the metaphor of the puzzle game. During the session of collective creation of meaning, the facilitator brings 5 or 6 briefs. This size constraint results from the fact that a brief is intended to be projected on a screen "(Sadok, and al., 2004). These briefs will be used as pieces of a puzzle to be built. Unlike the puzzle game, you do not have a model or all the pieces. "The Puzzle method consists of collectively building a puzzle (or several if necessary) on the screen of the work room, using forward-looking information as parts, on the one hand, and tacit suggestions and knowledge Which the participants then explain, on the other hand" (Blanco, S. and Lesca, N. 2003).

The Puzzle method is applied in the case of an indebted company to improve its performance, the search for performance results in a better competitiveness, but also gives a distribution of this performance. This distribution is important because optimizing the accounting process also means improving social cohesion, which ultimately constitutes intangible capital. In the context of finance, the adoption of a market efficiency hypothesis will advocate an assessment of the firm by the notion of economic risk for the purpose of assessing profitability over the long term. 
We can note that the accounting approach by the profit and loss account is a technique used preferentially by marketing. This, together with the fact that sales lead to the economies of scale of the company, is an additional reason for preferring marketing segmentation to any other to develop the company's strategy. No one can fully predict the value of the business over the long term. On the other hand, it is possible to evaluate to choose the corporate strategy or tactically decide the investments to be made by the financial analysis. Tactically, it would often be too expensive to carry out a full analysis of an investment if the resource at stake is not critical. It is then possible to make rapid financial analyzes generally by cash flow. Strategically, investment in critical resources must be analyzed in greater depth. It is possible to carry out an analysis by the strategic activity or by the harmonization of indicators that can be found in the services of the company. In this case, strategies will analysis the coverage of fixed costs by sales. The financial indicators following the same segmentation will indicate whether this represents an opportunity in terms of the financial value and the gain in synergy of intangible capital (Hached, R., 2014).

\section{Case study "Reorganization of the Accounting Process"}

The problem is posed to the company when there is a daily commute between the company's headquarters and the warehouse in which all customer records are stored, claims, refund claims, adjusted ... One wonders how many people were assigned to manage this enormous mass of financial data. It is for this reason, the Director decided to reorganize the accounting process. A collective reflection session of the steering committee was decided. The agenda is the following: "How to reorganize the accounting process?" (Figure 2). 




\section{Accounting}

Figure 2. Analysis of the accounting process

The person responsible for preparing the FULL financial documents that could be used at the next meeting of the Management Committee has collected 300 FULL financial documents which may correspond to the agenda, but it is out of the question that this person reads them all in the short time it has (data overload). This person will be named "facilitator" afterwards. 
To prepare for the meeting, the facilitator should perform the following tasks:

- search for and extract FULL financial documents closer to the agenda and likely to contain possible information of an anticipatory nature announcing changes in the business environment,

- answering about the degree of reliability of each of them,

- make numerous trips from one FULL financial document to another to accompany the participants in their reflections and interactions.

The Director has decided to make more frequent such meetings of work called "Collective Creation of meaning or $\mathrm{CCM}^{\prime}$ to interpret the information of an anticipatory nature. But it requires that the time of preparation and manipulations of the FULL financial documents are as short as possible to reduce the administrative costs, increase the reactivity and not hinder the reflections: without a significant time saving the day before will be abandoned (Blanco, S., and al. 2003).

The search for value data directly exploitable, in total adequacy with the information needs but also the financial, technical and human resources of the company, in order to optimize the time of decision making and catalyze the action, renders obsolete Any classification of information by nature. The criteria of effectiveness, utility and value must therefore prevail in any attempt, action or policy of management of the information. However, even if this approach is absolute, it remains interesting to separate formalized sources from digital sources as they offer advantages and have very different and complementary disadvantages. The following nine criteria are therefore found:

Ease of access to information: information that has a reduced access time and is accessible, the more your competitors can seize it, unless it is internal to the company. Likewise, the more accessible the information, the quicker your reaction must be so that it does not lose its relevance and interest.

The acquisition cost: The acquisition cost varies depending on the degree of formalization of the information. Thus, data represents a limited investment cost in the light of enriched, valued and worked information. Even if the cost of acquiring data is decisive, it is not enough, for two fundamental reasons: A part of data acquired results in induced costs that it was assessed a priori and not once the acquisition was made (Rouibah, K. 2003). As expensive as it is, information must correspond to the needs of the company, as well as to its financial, technical and human resources. Indeed, what is the good of acquiring a sophisticated database if your real needs are less and no one is able to exploit it?

Current criterion: The relevance and value of information is obviously linked to its "freshness", its actuality.

Reliability: Like any living organism, the company must avoid "swallowing" dubious foods.

To do so, before validating information whose unthinking use could threaten the company, it is necessary to evaluate its reliability.

Added value: The value of information is proportional to its contributions; or any information contains elements you already know! What is important is the novelty of certain elements that it conveys. In addition, a non-raw enriched data will be directly exploitable and the response time preceding the action will be reduced.

On the contrary, information with low added value requires a formalization work, a more or less long enrichment; thus your reaction will be less prompt and you risk being overwhelmed by the constant evolution of your environment.

Again, the more formalized the information, the less it requires treatment, valorization, to be directly operational. Moreover, the criterion of exploitability is inseparable from the criterion measuring the adequacy of the information sought for the needs of the company.

The induced costs: The acquisition of information is the first step in a long chain leading to decision-making. Thus, any data must be stored, enhanced, valued, formatted, purified, managed, before being used.

Uniqueness and exclusivity: When a decision-maker exclusively holds highly potential information, the entrepreneur has a strategic competitive advantage, provided of course to exploit this windfall quickly and efficiently. However, in order to assess the uniqueness of information, it must be compared with other sources that could replace it. The surprises can be great because it is often possible to replace a source that was believed unique by another offering equivalent or even superior characteristics.

Lifetime: Information is a perishable commodity, the longer it takes to use it, the more it is obsolete, cumbersome and expensive for the company. 
However, the lifetime of information also depends on its nature; thus a stock exchange hose is as ephemeral as a butterfly, whereas a substantial file resulting from a long process of formalization, such as a market study, offers a life of several weeks or even several months if it is accompanied by Term prevention (Rouibah, K., and al., 2002). Each of these criteria has strong correlations with others (Figure 3); however the major and omnipresent criterion is related to the notion of cost. These costs, if appreciated, managed and controlled, represent an investment and not an expense. Moreover, they foreshadow the future contribution and profitability of a true policy of strategic management of information. Whether the information is formalized or digital, the evaluation criteria developed above allow us to accurately scan the information. The analysis grid thus developed is an effective tool. It is possible to highlight the advantages and disadvantages of formalized information and digital data through the spectrum of information. It goes without saying that this formatting is at least "speaking" and can be used for all types of data and sources (Chapus, E., and al., 1998). The Grid of analysis of the accounting process is illustrated in figure 3.

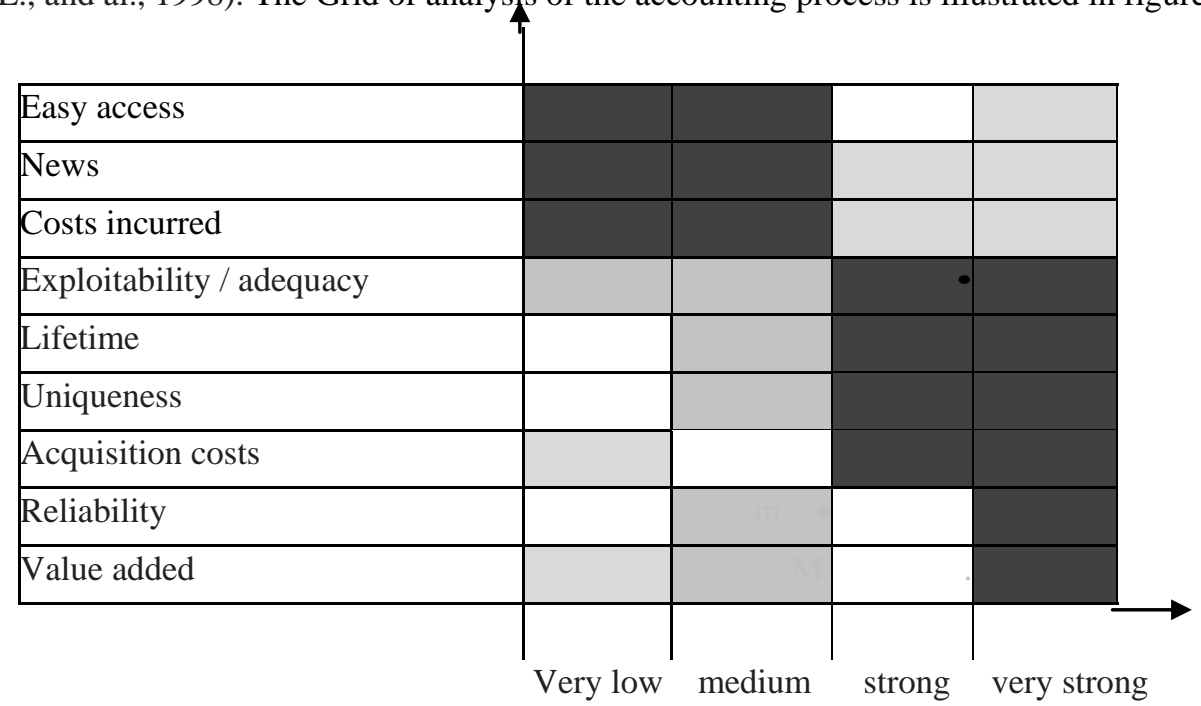

Figure 3. Grid of analysis of the accounting process

Table 1. List of criteria

\begin{tabular}{lll}
\hline Criteria & Formalized information & Digital data \\
\hline Easy access & Very low to medium & Strong to very strong \\
Acquisition costs & Very strong means & Very low to low \\
News & Very low to medium & Medium to very high \\
Reliability & Strong to very strong & Low to medium \\
Value added & Strong to very strong & Very low to medium \\
Exploitability / adequacy & Medium to very high & Very low to medium \\
Costs incurred & Very low to medium & Very strong means \\
Uniqueness & Medium to very high & Low to medium \\
Lifetime & Medium to very high & Very low to medium \\
\hline
\end{tabular}




\section{Result}

A collective reflection session, involving various directors, was decided. The agenda is worded as follows: "Reorganization of the accounting process". The facilitator has 300 FULL financial documents which can correspond to the agenda, but it is out of question that the facilitator reads them all in the short time that he has (information overload): the facilitator must therefore extract the most relevant and the number of these must not exceed the fortnight in order to be exploited at the meeting.

For the meeting, the facilitator must therefore put himself in condition to:

- present the selected FULL financial documents as visually as possible,

- respond rapidly to the demands that could be formulated, "on the run", the participants,

- accompany the progress of the interactions, without breaking the rhythm of these, between the participants by projecting the FULL financial documents possibly capable of helping the collective reflection,

- respond, as and when promptly, to possible questions such as: "Is this information reliable? Do we have information that would complement it? Do we have information that would contradict or disprove...? ".

It was in order to provide efficient support, to meet such conditions, that was designed. It was the subject of a first experiment for the case presented below. During the preparation of the next working session, scheduled for the next day, the first task of the facilitator is to discover the contents of the 300 FULL documents. It has very little time for that. Here are the rest of his operations. The animator observes three types of graphic forms: double arrows; small local constellations centered on their nucleus; arms of the local constellations, arms consisting of the sequences of FULL documents connected together by a single arrow.

Thanks to the properties of the neighborhood measure and the obtained graphs, it may suffice to read only the text of the nucleus to:

- decide to abandon the reading of all the FULL documents of the constellation

- be alerted on the usefulness of reading at least the FULL documents of the first crown of the nucleus: increased attention.

To begin, the animator clicks on a local constellation that we will note LC. He gets a page with the representation of the local constellation, a table with the cloud of LC words and a table containing the clouds of words for each arm of the constellation. LC's word cloud allows the facilitator to have a general idea of the topic addressed by LC. The word clouds of the branches give the animator an insight into how the subject of LC is approached by each branch (Lesca, H., and al., 1996).

\section{Conclusion}

The experimentation carried out shows that the hypothesis of research is validated: it encourages replicating the approach on the other processes. The aim was to help overcome the information overload caused by large volumes of digital data and, consequently, to reduce the administrative costs of informative early warning and cognitive overload. The experimentation carried out makes it possible to observe:

- Gain time processing and dissemination of information for the exploration of FULL financial data drawn;

- Facilitation to search for information that is reliable, or mutually reinforcing;

- A facilitation to suggest links between information in the working group responsible for interpreting weak signals.

\section{References}

ALMEIDA, F. C.; LESCA, H.; CANTON, A. W. P. (2016). Intrinsic motivation for knowledge sharing competitive intelligence process in a telecom company. Journal of Knowledge Management, 20(6), 1282-1301, 2016. https://doi.org/10.1108/JKM-02-2016-0083

BLANCO, S. et LESCA, H. (1997). Environmental scanning : designing a collective learning process to track down weak signals. Actes de la 3e Conférence de l'AIS Amérique (Association for Information Systems), Indianapolis, USA, 15-17 août 1997, pp.82-83.

BLANCO, S., CARON-FASAN, M-L., LESCA H. (2003). Developing capabilities to Create Collective Intelligence Within Organizations. The Journal of Competitive Intelligence and Management, JCIM, 1(2).

BLANCO, S. et LESCA, H. (1998). Business Intelligence : integrating knowledge into the selection of early warning signals. ECIS' Workshop on Knowledge Management, Edité par CEA/DIST, Saclay, France, 15p. 
BLANCO, S. et LESCA, N. (2003) - From weak signals to anticipative information : Learning from the implementation of an information selection method. C.E.R.A.G. umr CNRS 5820 n 2003-4, 15 p.

BASSET, H. (2013). De la veille à l'intelligence scientifique : définitions et concepts de base. In H. Basset (ed.), Maîtriser la veille pour l'intelligence scientifique, p. 10-13. Paris : Techniques de l'Ingénieur.

CHAPUS, E., LESCA, H., RAYMOND, L. (1998). Collective learning within an environmental scanning coalition of small regional firms : towards a modelization. CERAG, ESA, série recherche 98-15, 24 p.

DREVON, E., Maurel, D. et Dufour, C. (2016). Veille stratégique et veille informationnelle dans un contexte d'organisation durable. Dans Communication, information et savoir : quel management pour une organisation durable? (pp. 103-122). Montréal: Université de Moncton.

DELENGAIGNE, X, (2014). Organiser sa veille sur Internet : au-delà de Google... : outils et astuces pour le professionnel. Paris : Eyrolles. [Disponible à UdeM - BLSH : BSI (ZA 4230 D45 2014)]

DEISS, J. (2015). L'art de faire des recherches et de partager l'information : pratiques et techniques de veille et de curation sur Internet. Limoges : Éditions Fyp. [Disponible à UdeM - BLSH : BSI (ZA 4230 D447 2015)]

DE MONTMOLLIN, P. (2013). Utilité de la veille stratégiques au sein d'institutions culturelles: l'exemple du Musée d'histoire naturelle de Neuchâtel. (Mémoire de Certificat en gestion de documentation et des bibliothèques). Université de Fribourg, Neuchâtel (Suisse).

DE VINCENZO \& al., (2016). The Human Group Optimizer (HGO): Mimicking the collective intelligence of human groups as an optimization tool for combinatorial problems.

EMILIE VAYRE, (2016). Effets du travail en débordement via les TIC sur l'engagement au travail et l'interface travail / « hors travail » des cadres : rôle médiateur du rapport à Internet, XIX ème congrès de l'Association internationale de psychologie du travail, Bruxelles 10-13 Juillet 2016.

FREITAS, P.; FREITAS, H.; JANISSEK-MUNIZ, R. (2008). Tools for Anticipative Strategic Intelligence (ASI). In: 13eme AIM, 2008, Paris/França. Anais do 13eme AIM.

GALlO, J., MCGIVERN, M., WILliAMS, S. (2016). BI Experts' Perspective: Keep SaaS From Sinking Your BI Program. Business Intelligence Journal,| 21(39), 14.

GRIFFIN, C., R., SCOTT, M., GOLDEN, W. (2014). Exploring Factors that influence the Knowledge Worker in a Business environment.

HACHED, RAJA. (2014). Financial covenants and financial reporting conservatism: French evidence. International Journal of Basic and applied Sciences, 3(3).

KAMOUN-CHOUK, S. (2007). Environmental scanning in Tunisian Manufacturing SMEs. Journal of Information \& Knowledge Management, 6(1), 57-67. https://doi.org/10.1142/S0219649207001627

LAKHOUA M.N. (2018). The Need for systemic analysis and design methodology of the medical equipments, International Journal of Applied Systemic Studies, ISSN: 1751-0589, Inderscience, Vol.8, №1.

LAKHOUA M.N. (2013). "Systemic analysis of an industrial system: case study of a grain silo", Arabian Journal for Science and Engineering, ISSN: 1319-8025, Springer Publishing, Vol.38.

LESCA, H., LESCA, N. (2011). Weak Signals for Strategic Intelligence, Anticipation Tool for Managers. Wiley USA, 230 p. https://doi.org/10.1002/9781118602775

LESCA, H.; LESCA, N. (2014). Strategic Decisions and Weak Signals. Anticipation for Decision-Making. ISTE WILEY 2014. Chap 3.

LESCA, N. et CARON-FASAN, M.-L. (2008). Strategic Scanning Project Failure and Abandonment Factors: Lessons Learned. European Journal of Information Systems (EJIS), 17, September 2008, 371-386. https://doi.org/10.1057/ejis.2008.21

LESCA, N. CARON-FASAN, M.-L. et FALCY, S. (2008). How Managers Interpret Scanning Information?, Cahier de recherche du CERAG, $\mathrm{n}^{\circ} 2008-10,32$.

SADOK, M.; LESCA, H. (2007). A proactive scheme for the reduction of security costs and risks in virtual supply chain management, Cahier de Recherche ${ }^{\circ}{ }^{\circ}$ 2007-20, C.E.R.A.G. U.M.R.CNRS 5820, 15 p.

LESCA, H. (1996). Business Intelligence for strategic management : state of the issue and research program. CERAG, ESA, série recherche 96-14, 15 p. 
LESCA, H., CARON, M-L., TARDIF, F. (1996). Strategic observation : creating collective intelligence within the company. CERAG, ESA, série recherche 96-10, oct., 21 p.

LESCA, H., ROUIBAH, K. (1996). A proposal approach for strategic probe : a support structured scanning information. In Business Process Modelling, sous la direction de SCHOLZ-REITER, R. et STICKEL, E., Springer. Proceedings of the International Conference on Intelligent technologies in Human-relatd Sciences, pp.197-203.

LESZCZYNSKA, D., LESCA, H. (2004). STRATEGIC SCANNING: USEFULNESS OF " IN-THE-FIELD" INFORMATION FOR SMALL AND MEDIUM-SIZED BUSINESSES AND INDUSTRIES LOOKING FOR NEW OUTLETS PRODUCT/MARKET . Papier de Recherche CERAG 2004 - IAE Nice.

ROUIBAH, K. (2003). Environmental Scanning, Anticipatory Information and Associated problems : Insight from Kuwait. Communications of the International Information Management Association, 3(1).

ROUIBAH, K., OULD-ALI, S. (2002). PUZZLE: a concept and prototype for linking business intelligence to business strategy. Journal of Strategic Information Systems, 11, 133-152. https://doi.org/10.1016/S0963-8687(02)00005-7

ROUIBAH, K. et LESCA, H. (1997). A proposal approach for business intelligence : concept and prototype. Hawaii International Conference on System Science 30, january 7-10, Wailea, Maui, Hawaii.

ROUIBAH, K., LESCA, H., MALEK, S. (1997). Business intelligence : a processing heuristic for weak signal. 15th Annual international conference of Association of Management and International Association of Management (AoM/IAoM), Montréal, Québec, Canada août 6-9, in Proceedings supplemental, 15(1), 97-106.

ROUIBAH, K. et LESCA, H. (1996). Decision support for strategic probe. Advances in Industrial Engineering Applications and Pratices I, 342-349.

SADOK, M., LESCA, H. (2009). A Business Intelligence Model for SMEs Based on Tacit Knowledge. Communications of the IBIMA, 7(20).

SADOK, M., BENABDALLAH, S., LESCA, H. (2004). Collective Intelligence Process for risk reduction: theory, implementation and practical application to security incident response. Papier de Recherche CERAG (Grenoble - Iset'Com (Tunis) - ANCE (Tunis). 